\title{
Alain LERNOULD, Simplicius. Commentaire sur la Physique d'Aristote. Livre II, ch. 1-3
}

Introduction, traduction, notes et bibliographie par Alain Lernould, Presses universitaires du Septentrion, Lille, 2019 (Cahiers de Philologie), 234 p., ISBN : 2-7574-2465-3

\section{Philippe Soulier}

\section{(2) OpenEdition}

\section{Journals}

Édition électronique

URL : https://journals.openedition.org/philosant/4171

DOI : $10.4000 /$ philosant.4171

ISSN : 2648-2789

\section{Éditeur}

Éditions Vrin

\section{Édition imprimée}

Date de publication : 15 décembre 2021

Pagination : 298-301

ISBN : 978-2-7116-3036-3

ISSN : $1634-4561$

\section{Référence électronique}

Philippe Soulier, «Alain LERnould, Simplicius. Commentaire sur la Physique d'Aristote. Livre II, ch. 1-3», Philosophie antique [En ligne], 21 | 2021, mis en ligne le 16 mars 2021, consulté le 06 décembre 2022. URL : http://journals.openedition.org/philosant/4171; DOI : https://doi.org/10.4000/philosant.4171

\section{(c) $(1)$}

Creative Commons - Attribution - Pas d'Utilisation Commerciale - Pas de Modification 4.0 International - CC BY-NC-ND 4.0

https://creativecommons.org/licenses/by-nc-nd/4.0/ 
Alain Lernould, Simplicius. Commentaire sur la Physique d'Aristote. Livre II, ch. 1-3. Introduction, traduction, notes et bibliographie par Alain Lernould, Presses universitaires du Septentrion, Lille, 2019 (Cahiers de Philologie), 234 p., ISBN : 2-7574-2465-3.

Est-ce bien la nature elle-même qui doit être comprise comme la cause proprement productrice des corps naturels et de leurs changements, ou bien le régime de production dévolu à la causalité naturelle n'est-il qu'un adjuvant : l'instrument par lequel agit une causalité d'un tout autre ordre ? Dans le monumental Commentaire à la Physique d'Aristote que rédigea vers 540 le néoplatonicien Simplicius de Cilicie, cette question gouverne la partie consacrée aux trois premiers chapitres du livre II.

Alain Lernould en présente ici la première traduction française, sur la base du texte grec établi par H. Diels en 1882. Cette traduction est précédée d'une introduction synoptique aussi claire que concise (p. 11-35), qui traite tour à tour (1) de la date de composition des commentaires aristotéliciens de Simplicius, (2) de l'importance du livre II de la Physique d'Aristote, (3) du commentaire au livre II comme véritable introduction à la philosophie de la nature du Néoplatonisme tardif, (4) du lien entre physique et théologie, attesté par l'examen du but et de l'utilité de la Physique d'Aristote selon Simplicius, (5) de l'accord entre Aristote et Platon, (6) de la place d'Alexandre d'Aphrodise dans le commentaire de Simplicius à la Physique. Cette introduction est suivie d'un résumé analytique du texte traduit (p. 37-46) et d'une liste de 56 modifications apportées au texte grec de Diels (p. 49-51). Précisons qu'au fil de la traduction, ces corrections font chaque fois l'objet d'une mention spécifique dans une note appropriée, et sont souvent substantiellement

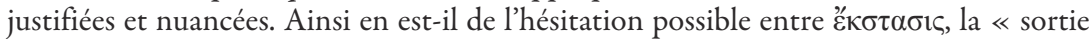

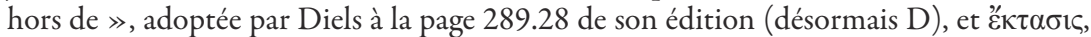
l'« extension », préférée par Lernould (L). La note 286 p. 127 recense les passages parallèles chez Simplicius et indique des variations similaires dans des textes de Proclus et de Plutarque, et d'autres occurrences chez Damascius. Par ailleurs, à la différence de Diels, l'auteur donne dans le corps du texte les lemmes complets de la Physique tels que Simplicius les lit (en indiquant en note, le cas échéant, les écarts entre le texte d'Aristote dans Simplicius et celui dans les éditions modernes de référence). L'auteur prend aussi soin de corriger, lorsque cela lui paraît nécessaire, le découpage des lemmes opéré par Diels (e.g. p. 100, n. 191 L, ad 277.10 D ; p. 170, n. 411 L, ad 310.18 D). La traduction elle-même est encadrée par de sobres intertitres à la typographie soignée, qui facilitent sa lecture. Les notes de bas de page sont précises mais sans inflation. Viennent enfin une bibliographie, un abondant index des termes grecs (p. 219-231) et un index des auteurs cités par Simplicius.

Sur le plan doctrinal, les précédents travaux de l'auteur sur les liens entre physique et théologie dans le néoplatonisme tardif, en particulier dans l'exégèse proclusienne du Timée, lui permettent de mettre en évidence, tant dans l'introduction que dans les notes, les enjeux philosophiques du commentaire simplicien, notamment dans la manière dont il intègre le commentaire d'Alexandre d'Aphrodise, l'autorité majeure avec laquelle Simplicius discute. L'auteur reprend à son compte la thèse de M. Rashed selon laquelle le commentaire de Simplicius est le commentaire d'Alexandre réécrit par Simplicius, dans la mesure où le centre de gravité de l'aristotélisme est le monde sensible : Alexandre apparaît alors comme « le porte-voix du Sensible bien compris » (M. Rashed, 2011, Alexandre d'Aphrodise, Commentaire perdu à la Physique d'Aristote (Livres IV-VIII). Les scholies byzantines, Berlin). Néanmoins, précise l'auteur, « il ne faudrait pas penser que le commentaire de Simplicius sur la Physique d'Aristote est, pour Simplicius, plus le 
commentaire d'un commentaire (celui d'Alexandre) qu'un commentaire sur Aristote lui-même. C'est bien Aristote qu'il s'agit pour lui de restituer dans toute sa vérité, c'est-à-dire un Aristote allié de Platon » (p. 35). À l'instar de Proclus, commentateur du Timée de Platon, Simplicius en tant que commentateur de la Physique d'Aristote se pose en ardent défenseur d'une physique théologique, puisque selon la philosophie néoplatonicienne de la Nature, celle-ci est logos, un logos physique émanant de logoi mathématiques, eux-mêmes images des Formes intelligibles. Le sensible est ainsi fondé dans l'intelligible par une physique proprement «logique » (ibid.).

Toutefois il s'agit pour Simplicius d'établir cela patiemment, à travers l'exégèse même du texte de la Physique. Ainsi argumente-t-il contre l'idée d'Alexandre pour qui Aristote, en définissant la nature comme principe du mouvement, définit aussi l'âme (268.18269.4 D, p. 75 L). Or Simplicius n'accepte pas d'attribuer à Aristote une telle assimilation de la nature avec l'âme. Dès le début du commentaire du ch. 1, il s'attache à expliquer pourquoi Aristote dit que les animaux et les plantes sont « par nature » (Phys. 192b910), alors que c'est une âme qui semble caractériser ces êtres (In Ph. 262.13-263.30 D, p. $61 \mathrm{~L}$ ). L'enjeu doctrinal de ce qui paraît d'abord n'être qu'une argutie exégétique s'éclairera lorsqu'au début du commentaire du ch. 2, dans le cadre d'une récapitulation générale sur ce qu'est la nature, Simplicius introduira la tripartition néoplatonicienne du réel en étants supra-naturels, naturels, infra-naturels (285.30-289.35 D, p. 119-128 L). Comme le souligne l'auteur, la triade sensible où l'on s'élève de la matière à la forme en passant par la nature est le reflet inversé de la triade intelligible qui procède de l'Être à l'Intellect en passant par la Vie. On comprend dès lors en quoi Simplicius prête à la physique la vertu anagogique de nous faire remonter du Sensible jusqu'à l'Âme et de là jusqu'aux Formes dans l'Intellect divin, i.e. le Démiurge du Timée assimilé au premier moteur d'Aristote (p. 23).

Mais cela implique, justement, que l'on distingue d'abord entre la nature et l'âme, y compris dans la partie inférieure de celle-ci, l'âme végétative avec laquelle on l'identifie souvent, alors même que pour Aristote elle est autre chose (286.20-288.32 D, p. 120-125 L). En effet l'âme est principe du mouvoir, tandis que la nature est principe de l'être-mû. La hiérarchie des causes efficientes : Intellect et Âme, conduit à concevoir la Nature comme une cause instrumentale qui à la fois est mue par une autre chose et meut une autre chose (317.6-28 D, p. $187 \mathrm{~L})$. Seul « l'Intellect tant honoré » (ó $\pi \mathrm{o} \lambda v \tau i ́ \mu \eta \tau o \zeta$ vôิৎ, expression néoplatonicienne courante) est proprement cause efficiente ou productrice du mouvement, dans la mesure où il est strictement moteur et non pas mû, à la différence de l'Âme auto-mobile. Et lorsque Aristote qualifie l'âme d' « immobile » (dans le De Anima), il le fait uniquement, selon Simplicius, « en jugeant que seules sont mues les choses qui changent sous le mode corporel » (317.19 D). Toutefois c'est bel et bien à Platon que Simplicius rapporte sa caractérisation de l'Âme comme mue par soi (318.12-13 D).

La question de la causalité forme ainsi l'horizon philosophique du commentaire de ces trois chapitres, et c'est dans l'exégèse du troisième que Simplicius livre la doctrine des causes, anticipée dès le grand Prologue de l'œuvre. Celles que le Cilicien désigne chez les Péripatéticiens sont à la fois les deux causes réellement causes ou «proprement dites », i.e. les causes productrice et finale, et aussi les deux causes auxiliaires ou accessoires ( $\tau \dot{\alpha}$

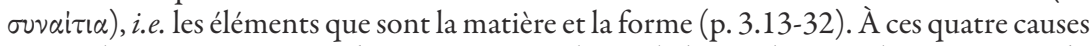
aristotéliciennes s'ajoutent deux autres, complétant la hiérarchie néoplatonicienne : du côté des vraies causes, la cause paradigmatique ( $\tau$ ò $\pi \alpha \rho \alpha \delta \varepsilon \imath \gamma \mu \alpha \tau$ เòv, le modèle) et du côté des causes auxiliaires, la cause instrumentale, tò ỏ pyavıkóv. 
À partir de là peut être située la Nature : « il est bon de dire que la nature est plutôt cause accessoire (бuvaítıৎ), tandis que sont causes productrices de la génération et de la corruption les mouvements des corps célestes, dont les choses d'ici-bas subissent l'influence; que sont des causes plus élevées les raisons psychiques de [ces] mouvements, et plus haut encore, les Formes intellectives; et c'est à partir de ces Formes premières que

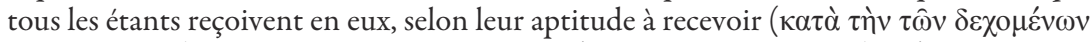

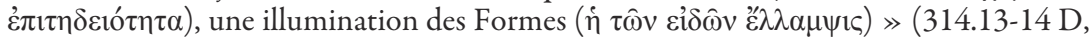
p. $180 \mathrm{~L}$. On notera la prégnance du vocabulaire proclusien dans ce passage). Dans une belle formule, Lernould affirme que « le commentaire donne à Simplicius l'occasion d'élévations spirituelles qui sont comme des déchirures dans un ciel sombre par lesquelles on entrevoit, dans un trait de lumière, toute la splendeur des hiérarchies intelligibles et divines $\gg$ (p. 23).

En tant que principe du mouvement, la Nature est « en quelque sorte cause productrice prochaine » des étants naturels (Simplicius, $3.26 \mathrm{D}$ ). Néanmoins elle n'est pas cause productrice au sens propre du terme, parce qu'elle est une cause immanente (selon Aristote, « la nature réside dans un substrat », Phys. 192b34). Au contraire, une cause proprement productrice transcende nécessairement son produit, en vertu du grand principe proclusien : « tout être qui en produit un autre est supérieur à son produit » (Proclus, Éléments de Théologie, \$7, plusieurs fois rappelé par l'auteur, e.g. p. 124 n. 277, p. 172 n. 7). Simplicius le retranscrit ainsi : « ce qui réside dans un substrat ne sera pas au sens strict un principe qui meut le substrat » (p. 287.29-30 D).

Quant à la mobilisation de la thèse platonicienne selon laquelle les Formes sont les paradigmes des réalités naturelles, elle constitue un autre point nodal de la confrontation philosophique et exégétique avec Alexandre, car selon ce dernier, la forme naturelle qu'Aristote appelle « modèle », $\pi \alpha \rho \alpha ́ \delta \varepsilon 1 \gamma \mu \alpha$, en 194b26, n'est pas modèle en tant qu'Idée. À cette occasion, Simplicius présente une longue et très intéressante citation du commentaire d'Alexandre (310.20-311.37 D, p. 170-175 L). C'est un développement embryologique inspiré du traité De la génération des animaux d'Aristote : l'enchaînement automatique des mouvements imprimés par le sperme du mâle déposé dans la matière qu'offre la femelle est comparé avec le mécanisme des automates. La semence est à la fois cause matérielle, productrice et motrice, la communication du mouvement étant une forme de production. Mais Simplicius critique ce modèle embryologique, et cela touche le centre même de la philosophie de la nature qui gouverne son commentaire : en quel sens la forme immanente qui advient peut-elle être modèle, et en quel sens la nature est-elle puissance irrationnelle ? Alexandre s'appuie sur le fait que la finalité n'implique pas forcément la délibération. La forme est une sorte de terme, elle se présente comme un but auquel vise la nature, et est pour cette raison appelée « modèle »; mais la nature n'œuvre pas ici selon un choix ou une raison qui serait en elle, c'est pourquoi Aristote

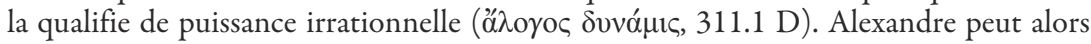
conclure : « la forme est donc modèle parce que la nature a incliné vers elle, non par choix, mais plutôt comme le font les automates $\gg$. Simplicius reconnaît comme évident qu'Aristote n'appelle pas ici la forme « modèle » au sens d'Idée, mais simplement comme élément advenu du composé : ce n'est donc pas l'agent producteur (

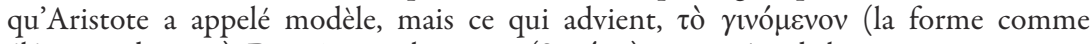

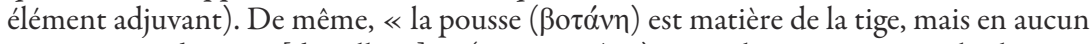
cas cause productrice [de celle-ci] »(312.33-34 D). Dans la concaténation biologique des causes, les choses qui précèdent sont plutôt causes matérielles que productrices et seules sont modèles au sens propre les Formes dans l'Intellect (314.15-24 D). Quant à la caractérisation de la nature comme puissance irrationnelle, pour Simplicius il ne 
peut y avoir ni ordre ni fin déterminée dans la production si le producteur n'a pas la connaissance ; toutefois celui-ci n'est pas la nature.

On le voit, la Physique est clairement lue par Simplicius comme déployant une philosophie de la nature néoplatonicienne, dont le commentaire livre de nombreux éléments essentiels; mais comme le rappelle l'auteur, il reste « qu'il ne faut pas réduire le commentaire à un simple corps de doctrine philosophique (...), le contenu philosophique ne peut être dissocié des dimensions exégétique, pédagogique et proprement spirituelle, qui constituent l'âme même du commentaire » (p. 18).

Philippe Soulier

Université de Nantes

Magna voce. Effets et pouvoirs de la voix dans la philosophie et la littérature antiques, sous la direction d'Anne-Isabelle Bouton-Touboulic, Paris, Classiques Garnier, 2021 (Kaïnon - Anthropologie de la pensée ancienne, 19), 473 p., ISBN : 978-2-406-10660-9.

En partie issu d'un colloque sur les «Effets de voix : la voix et les voix dans la pensée et la littérature romaines » qui s'est tenu en novembre 2014 à Lille, ce livre rassemble dix-huit contributions, classées selon quatre grandes catégories : I. Philosophie, II. Rhétorique et médecine, III. Acoustique, théâtre et littérature, IV. Linguistique, anthropologie, religion. Il comprend également une bibliographie (p. 385-423), un index des noms (p. 425-429), un index des passages cités (p. 431-457), un index des notions (p. 459-463) ainsi que le résumé de chaque essai (p. 465-470).

Un rapide survol de la table des matières et la lecture des premières lignes de cet ouvrage pourraient susciter de prime abord deux problèmes : $1^{\circ}$ nulle part l'éditrice ne définit strictement ce qu'elle entend par la « voix » qu'elle met au centre de son livre et qu'elle a choisie pour lui donner son titre, Magna Voce; $2^{\circ}$ les contributions qui y sont rassemblées obéissent somme toute à un classement très académique, qui ne semble pas remettre en question le cloisonnement artificiel entre les disciplines qui a si longtemps caractérisé le monde académique.

Mais en réalité, il n'en est rien.

Tout d'abord, Anne-Isabelle Bouton-Touboulic revendique l'idée qu'il s'agit ici d'appréhender « la voix comme une phénomène total, qui se diffracte en de nombreux domaines (musical, poétique, rhétorique, linguistique, philosophique, médical et religieux...) » (p. 9). C'est précisément ce dont il faut se réjouir, car c'est ce qui fait toute la force, toute la richesse, et tout l'intérêt de cet ouvrage. Les essais qui le composent parviennent en effet à créer de nouveaux réseaux de sens, à rassembler des approches particulières, à comparer des perspectives et des méthodes diverses. En invitant chaque contributeur à interpréter et définir le problème de la « voix » en fonction des impératifs propres à son domaine de spécialité, Anne-Isabelle Bouton-Touboulic a rendu possible le surgissement de questions et de méthodes empruntées à l'anthropologie, à l'histoire des religions, à la philosophie, à la linguistique, à l'analyse littéraire ou théâtrale - chacune de ces approches n'en excluant jamais une autre.

Ensuite, cette profusion de thématiques, de problématiques, de discussions qui naissent autour de l'idée de la « voix », ne débouche pas sur l'organisation artificielle de contributions hétérogènes, qui seraient paresseusement rangées selon les champs disciplinaires dont elles relèvent. Bien au contraire : derrière un classement thématique 